\title{
Isolation of Eggerthella lenta from A Cutaneous Abscess Formed at Post- trauma Suture Site
}

\author{
Kavita Vijay Chaudhari ${ }^{1}$, Rakhi Biswas ${ }^{1}$, Meghna $C^{1}$, Sujatha Sistla ${ }^{1}$, Kadambari Dharanipragada ${ }^{2}$, \\ Balasubramanian Krishnan ${ }^{3}$, Akhilesh $\mathrm{R}^{3}$ \\ 'Jawaharlal Institute of Postgraduate Medical Education and Research, Department of Microbiology, Puducherry, \\ India \\ ${ }^{2}$ Jawaharlal Institute of Postgraduate Medical Education and Research, Department of Surgery, Puducherry, India \\ ${ }^{3}$ Jawaharlal Institute of Postgraduate Medical Education and Research, Dentistry, Puducherry, India
}

\begin{abstract}
Eggerthella lenta is an emerging pathogen. Its laboratory identification is difficult and hence only limited data is available on its disease spectrum. We report an occurrence of a polymicrobial infection in a cutaneous abscess formed at post-trauma suture site, with rare isolation of this anaerobe, in a healthy immunocompetent man. $J$ Microbiol Infect Dis 2020; 10(4): 230-233.
\end{abstract}

Keywords: Eggerthella lenta, wound infection, anaerobic bacteria

\section{INTRODUCTION}

Eggerthella lenta, (formerly called Eubacterium lentum) is a non-sporulating Gram-positive anaerobic bacillus belonging to Coriobacteriaceae family [1]. Skin can serve as a rare source of this organism. The obstacles with laboratory identification of this organism, has resulted in sparse data on the disease spectrum. Here, we have described the isolation of this rare organism as part of a polymicrobial infection in a cutaneous abscess formed at posttrauma suture site, in a healthy immunocompetent man.

\section{CASE REPORT}

A 38-year-old male patient with history of road traffic accident was referred to our tertiary care hospital for definitive management, after preliminary treatment at a primary health care facility. He gave a history of loss of consciousness for approximately 5 minutes, not associated with vomiting or seizures. History of oral bleeding was present. There was no history of other comorbidities. The patient was conscious, oriented, cooperative and vitals were within normal limits. A maxillofacial extra oral sutured laceration was present over the left body of mandible extending to the neck region and a sutured lacerated wound was present on the left thigh. There was no obvious discharge or bleeding from both the wound sites at time of presentation. On investigation, a computerized tomography scan of the head detected comminution and soft tissue laceration on the left side of mandible extending to left body and angle ramus region. Complete hemogram done at time of admission was within normal limits.

Open reduction and internal fixation of the mandibular ramus fracture was performed. On post-operative day 2 , he had mild induration and slight tenderness at the surgical site over the thigh wound, which persisted even on the third day. Three sutures were removed from the thigh wound and 10-12 $\mathrm{ml}$ of pus was drained. Minimal debridement was done with surgical wash. Tissue bit was sent for both aerobic and anaerobic culture and antibiotic susceptibility testing. Patient was started on intravenous ceftriaxone $1 \mathrm{gram}$ twice daily. Aerobic cultures grew methicillin resistant Staphylococcus aureus (MRSA) sensitive to vancomycin and linezolid, and Enterobacter species sensitive to amikacin,

Correspondence: Dr. Rakhi Biswas, Jawaharlal Institute of Postgraduate Medical Education and Research, Department of Microbiology, Puducherry, India

Email: dr.rakhibiswas@gmail.com

Received: 21 December 2019 Accepted: 04 September 2020

Copyright (C JMID / Journal of Microbiology and Infectious Diseases 2020, All rights reserved 
gentamicin, meropenem, ceftazidime, ceftriaxone and ciprofloxacin. Gram stain of the smear prepared from the Robertsons' cooked meat broth containing the tissue, revealed nonsporting gram-positive rods as shown in Figure 1A. Colonies obtained on blood agar incubated anaerobically, in an anoxomat anaerobic jar were moist hemolytic speckled colonies (Figure1B). Aerotolerance was performed and it demonstrated the colony as that formed by an obligate anaerobe. Gram stain from the colony showed non-sporting gram-positive rods. Phenotypically, using the conventional biochemical tests, the given isolate was found positive for catalase; hydrogen sulphide formation, nitrate reduction and arginine hydrolysis. The above findings were also confirmed by MALDI-TOF MS (Vitek MS, bioMerieux, France) with a confidence value of 99.9 percent. The organism was identified as Eggerthella lenta. Susceptibility testing for this anaerobic isolate was not performed as it did not grow in subsequent cultures. The patient was treated with a regimen of tablet linezolid $600 \mathrm{mg}$ twice a day along with metronidazole $400 \mathrm{mg}$ thrice a day and tablet ciprofloxacin $500 \mathrm{mg}$ twice a day. He was given daily active wound care with debridement and dressing. The repeat aerobic and anaerobic cultures were sterile. At discharge, both the thigh and mandibular wound showed healthy granulation tissue. The patient was advised to continue same regimen for seven days along with proper wound care at the local hospital.
Anaerobic infections are usually polymicrobial in nature comprising both facultative and obligate anaerobes. In the present case, patient gave history of road traffic accident, and such accidental injuries usually are contaminated with soil and more likely to develop a polymicrobial infection. In this case, three pathogens were isolated. Although MRSA and Enterobacter species are known pathogens, $E$. lenta is an evolving pathogen gaining recognition in recent years.

Literature review has highlighted most of the reports on $E$. lenta from bacteremic cases, source being the GIT or skin infection, which has prolonged hospital stay of patients [2-4]. Malignancies, decubitus ulcers, Crohn's disease, appendicitis, pelvic inflammatory disease are few of the several conditions, with which this anaerobic microorganism has been associated [4]. Very few case reports have described isolation of $E$. lenta from infected wound $[5,6]$ Venugopal et al have described eight cases of infected decubitus ulcers that have caused $E$. lenta bacteremia. Two of these cases have been polymicrobial [6]. In the present case, we have a polymicrobial wound infection. A few reports have described its isolation from abscesses in the genitourinary tract and intra-abdominal locations [2]. More rarer presentations such as frontal sinusitis, pyomyositis, spondylodiscitis [3] and disseminated infection [4] have also been described (Table 1).

\section{DISCUSSION}

Table 1: Previously reported cases of E. lenta.

\begin{tabular}{lccc}
\hline Authors & Diagnosis & Organism isolated & Specimen \\
\hline Elias et al [9] & Multiple pyogenic liver abscess & E. lenta & Liver aspirate \\
Lattuada et al [5] & Cutaneous abscess in drug abuser & E. lenta & Abscess \\
Salameh et al [10] & Disseminated infection & E. lenta & Blood \\
Bok et al [11] & Spondylodiscitis & E. lenta & Bone aspirate \\
Urban et al [12] & Sinusitis & E. lenta & Aspirate \\
Palomino-Nicás & Pyomyositis & Eubacterium lentum and & Pus
\end{tabular}




et al [13] Streptococcus constellatus

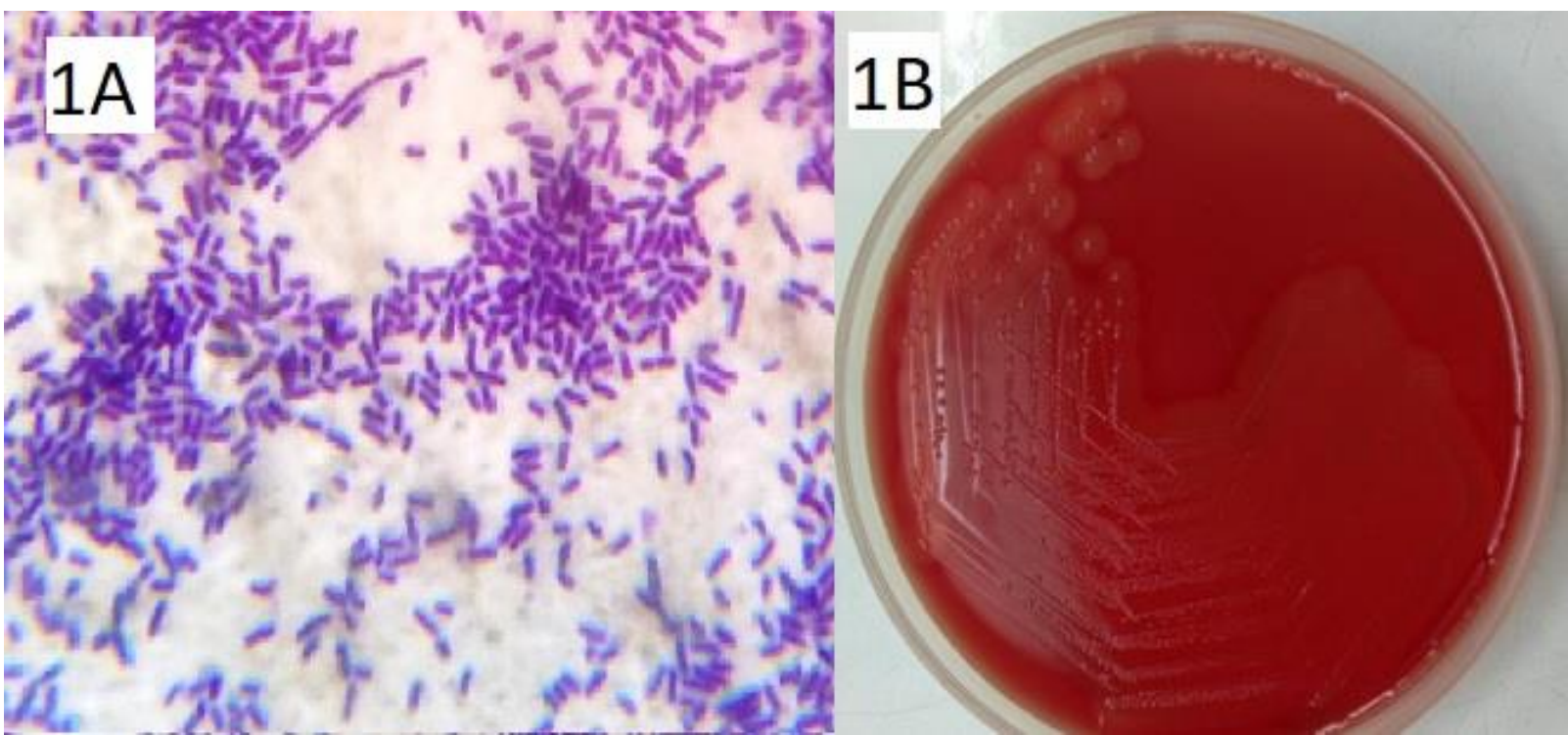

Figure 1A- Gram stain morphology of Eggerthella lenta - non-sporting gram positive rods.

Figure 1B- Colony morphology of Eggerthella lenta -moist hemolytic speckled colonies.

Among the virulence factors which are under investigation include inactivation of drugs like digoxin [7] by E. lenta. Stinear et al [8] identified presence of vanB locus in E.lenta isolated from faeces and also demonstrated the acquisition of this gene by it. They also hypothesized this organism to be the origin of vancomycin resistant enterococci (VRE)[8]. In our case, we identified $E$. lenta from tissue, however blood cultures could not be done, hence it is difficult to comment on source of infection. Also, the isolation of known pathogens such as MRSA and Enterobacter species make it difficult to decide on the pathogenic role of $E$. lenta. However, in the case report described by Lattauada et al, $E$. lenta was isolated as sole pathogen from cutaneous abscess in an injection drug user [5].

16sRNA sequencing and MALDI-TOF MS are the newer, rapid, confirmative methods for identification compared to phenotypic methods. Antimicrobial susceptibility for $E$. lenta is determined using minimum inhibitory concentration breakpoints, which could not be performed in this case, due to lack of subsequent growth. An intravenous beta-lactam antibiotic with or without metronidazole is the usual line of management for Eggerthella infections [4]. In cases of disseminated infections, Gardiner et al [4] have reported mortality ranging $36-43 \%$, however in absence of any other co-morbidities, infection usually responds to treatment as seen in the present case.

E. lenta is a delicate, slow growing organism and is difficult to identify in polymicrobial infections. This case report is an effort to highlight importance of carefully performed microbiological cultures for isolation of such fastidious organisms, a high clinical suspicion while treating such cases and also adding to the spectrum of disease caused by it.

\section{ACKNOWLEDGMENTS}

Conflict of interest: The authors declare no personal or financial conflict of interest.

Financial Disclosure: No financial support was received. 


\section{REFERENCES}

1. Eggerth $\mathrm{AH}$. The Gram-positive Non-spore-bearing Anaerobic Bacilli of Human Feces. J Bacteriol 1935; 30(3):277-99.

2. Brook I, Frazier EH. Significant recovery of nonsporulating anaerobic rods from clinical specimens.Clin Infect Dis Off Publ Infect Dis Soc Am 1993; 16(4):476-80.

3. Gardiner BJ, Korman TM, Junckerstorff RK. Eggerthella lenta bacteremia complicated by spondylodiscitis, psoas abscess, and meningitis. J Clin Microbiol. 2014 Apr; 52(4):1278-80.

4. Gardiner BJ, Tai AY, Kotsanas D, et al. Clinical and microbiological characteristics of Eggerthella lenta bacteremia. J Clin Microbiol 2015; 53(2):626-35.

5. Lattuada E, Zorzi A, Lanzafame M, et al. Cutaneous abscess due to Eubacterium lentum in injection drug user: a case report and review of the literature. $\mathrm{J}$ Infect 2005; 51(2):E71-72.

6. Venugopal AA, Szpunar S, Johnson LB. Risk and prognostic factors among patients with bacteremia due to Eggerthella lenta. Anaerobe 2012;18(4):4758.

7. Haiser HJ, Seim KL, Balskus EP, Turnbaugh PJ. Mechanistic insight into digoxin inactivation by Eggerthella lenta augments our understanding of its pharmacokinetics. Gut Microbes 2014; 5(2):233-8.

8. Stinear TP, Olden DC, Johnson PD, Davies JK, Grayson ML. Enterococcal van B resistance locus in anaerobic bacteria in human faeces. Lancet 2001 ; 357(9259):855-6.

9. Elias RM, Khoo SY, Pupaibool J, Nienaber J-H, Cummins NW. Multiple Pyogenic Liver Abscesses Caused by Eggerthella lenta Treated with Ertapenem: A Case Report. Case Rep Med 2012; 2012:718130.

10. Salameh A, Klotz SA, Zangeneh TT. Disseminated Infection Caused by Eggerthella lenta in a Previously Healthy Young Man: A Case Report. Case Rep Infect Dis 2012; 2012:517637.

11. Bok CW, $\mathrm{Ng}$ YS. Eggerthella lenta as a cause of anaerobic spondylodiscitis. Singapore Med J 2009; 50(12):e393-396.

12. Urban E, Gajdacs M, Torkos A. The incidence of anaerobic bacteria in adult patients with chronic sinusitis: A prospective, single-centre microbiological study. Eur $\mathrm{J}$ Microbiol Immunol 2020; 10(2):107-14.

13. Palomino-Nicás J, González E, Arroyo A, Cañas E, Hernanz W, Jerónimo P. Pyomyositis due to Eubacterium lentum and Streptococcus constellatus from a Periodontal Source. Clin Infect Dis 1996; 22(1):176-8. 